\title{
Effects of Repetitive Altitude Training on Salivary Immunoglobulin A Secretion in Collegiate Swimmers
}

\author{
Koichi Watanabe ${ }^{a}$, e, Subrina Jesmin ${ }^{a}$, Yosuke Murase ${ }^{b}$, Tsuyoshi Takedac ${ }^{c}$, \\ Takahisa Shirakid ${ }^{\mathrm{d}}$, Yasuo Sengoku ${ }^{\mathrm{a}}$
}

\begin{abstract}
Background: Altitude training has often been conducted just before main competition games in many sports. An increase in the frequency of upper respiratory tract infections and gastrointestinal infections due to an altitude-induced suppression of the immune system has been reported after altitude training. Salivary secretory immunoglobulin A ( $\mathrm{SIgA}$ ) is the major immunoglobulin of the mucosal immune system. A suppressive effect of heavy training on SIgA has been reported. However, little is known regarding the effects of repetitive altitude training and hypoxic exposure on SIgA. The objective of this study was to evaluate the changes in SIgA in swimmers undergoing repetitive altitude training at $1,900 \mathrm{~m}$.
\end{abstract}

Methods: Nine collegiate swimmers who experienced their first altitude training experience (FT group) were compared to nine swimmers who experienced repetitive training (RT group) and non-training subjects (Con group). Saliva was collected before ascent and eight times every 2 days during altitude training. SIgA levels were measured by enzyme-linked immunosorbent assays.

Results: Compared to the Con group, SIgA levels and the secretion velocity were decreased after ascent and were slowly restored in both the FT and RT groups. The chronological trends in SIgA levels were similar, even though the decline in SIgA levels in the FT group was larger than that in the RT group.

Conclusion: Altitude training and experience with altitude training may be one of the factors influencing SIgA.

Keywords: Salivary secretory immunoglobulin A; Altitude training; Experience; Swimming

Manuscript submitted May 25, 2019, accepted July 8, 2019

${ }^{a}$ Faculty of Health and Sport Sciences, University of Tsukuba, Ibaraki, Japan ${ }^{b}$ Sports and Physical Education Center, University of Tsukuba, Ibaraki, Japan 'Faculty of Sport Sciences, Waseda University, Saitama, Japan

${ }^{\mathrm{d} C o a c h i n g}$ Course, Department of Athletic Sport, Biwako Seikei Sport College, Shiga, Japan

${ }^{e}$ Corresponding Author: Koichi Watanabe, Faculty of Health and Sport Sciences, University of Tsukuba, 1-1-1, Tennodai, Tsukuba, Ibaraki 305-8575, Japan. Email: watanabe.koichi.ga@u.tsukuba.ac.jp

doi: https://doi.org/10.14740/jocmr3884

\section{Introduction}

Altitude training has been conducted in athletes since the 1960s in order to enhance the oxygen delivery to their peripheral tissue and to improve their aerobic capacity due to the erythropoiesis associated with acclimatization. Currently, the training is applied as a general training with the aim of enhancing aerobic capacity playing an important role in the physical preparation of athletes around the world $[1,2]$. Among athletes of competitive sports, altitude trainings are often done not only just before competitive events, but also during training periods in order to attain higher performances. Therefore, altitude training is an option in the annual swim training program among Japanese top elite swimmers, and it is not unusual that swimmer undergoes altitude training several times within a training season. Indeed, swimmers are amongst those athletes who use altitude training most often $[3,4]$.

Athletes can attain oxygen loads that they cannot get at sea level through altitude training. However, some researchers have suggested that the frequency of symptoms of the common cold or flu, such as rhinorrhea, nasal discharge, sore throat and gastrointestinal symptoms, such as diarrhea, are relatively high in athletes after altitude training $[1,5]$. These symptoms can easily lead to deterioration in their conditions and performances. Furthermore, symptoms that are similar to those of high mountain sickness are commonly observed as part of the infection-related symptoms, and, thus, it is often difficult to distinguish between infection and high mountain sickness. Bailey et al [1] suggested that an increase in stress hormones, such as cortisol, is one of the factors resulting in the deterioration in their conditions, but little is known about the impact of altitude training on the immune system. It is important that swimmers maintain their physical condition during high altitude training, which is important for enhancing performance at competitive events, and if one swimmer undergoes altitude training several times, the interval between the altitude trainings and/or the intensity of the second training are the cornerstones for making the training successful and safe.

Salivary secretory immunoglobulin A ( $\mathrm{SIgA})$ is the major immunoglobulin of the mucosal immune system. The suppressive effect of heavy training on mucosal immunity and the effect of exercise on SIgA secretion have been documented. However, little is known regarding the influence of hypoxia and repetitive hypoxic exposure on SIgA.

Thus, the aim of this study was to examine the effects of 
Table 1. Characteristics of the Study Groups

\begin{tabular}{llll} 
& FT $(\mathbf{n}=9)$ & RT $(\mathbf{n}=9)$ & Con $(\mathbf{n}=\mathbf{5})$ \\
\hline Age (years) & $19.6 \pm 1.2$ & $20.3 \pm 1.1$ & $24.6 \pm 3.6^{*, * *}$ \\
Height $(\mathrm{m})$ & $1.69 \pm 0.05$ & $1.73 \pm 0.09$ & $1.69 \pm 0.06$ \\
Weight $(\mathrm{kg})$ & $64.1 \pm 4.1$ & $64.9 \pm 9.4$ & $67.6 \pm 11.0$ \\
\hline
\end{tabular}

Values were expressed as mean $\pm \mathrm{SD}$. ${ }^{*} \mathrm{P}<0.05$ between $\mathrm{FT}$ and Con ${ }^{* *} \mathrm{P}<0.05$ between RT and Con. SD: standard deviation; FT: first altitude training; RT: repetitive training; Con: control.

altitude swim training on SIgA levels and the differences in the SIgA secretion after repeated experiences with hypoxic exposure.

\section{Materials and Methods}

\section{Subjects and methods}

We conducted the study in Kunming, China. Kunming is located 1,900 $\mathrm{m}$ above sea level, and the atmospheric air pressure is decreased to $80 \%$ of that at sea level. The first altitude training camp was held for 17 days in December. Nine collegiate swimmers (male/female $=7: 2$ ) joined the first training camp. They were classified as the first training experience group (FT group). Five healthy subjects who accompanied the swimmers joined this study as the control group (Con group). The second altitude training camp was held for 21 days in the next December. The third altitude training was conducted 24 days after the descent of the second training. Nine collegiate swimmers (male/female $=4: 5)$ joined the third training camp. They were assigned to the repetitive training group (RT group). Table 1 shows the characteristics of each group. The training loads of the first and third training camps are shown in Figure 1.

\section{Sampling and analysis}

Salivary samples were taken before ascent (day 0 ) and eight times every 2 days during the training camp (day 2 to day 16). After an overnight fast, saliva was collected from each subject using Salivette (Sarstedt AG \& Co., Numbrecht, Germany) after a 5-min rest in the morning. Every subject chewed a swab of Salivette every second for $1 \mathrm{~min}$. The Salivette swabs were then centrifuged in order to extract saliva. The extracted saliva was weighed, and the salivary secretion rate (SSR) was determined. After the anti-secretory component antibody and the anti-IgA antibody were added to the collected saliva, enzyme-linked immunosorbent assays that specifically detected SIgA were used to determine the SIgA concentrations (SIgA-C). The SIgA secretion velocity (SIgA-V) was calculated as follows: SIgA-V $(\mu \mathrm{g} /$ $\min )=$ Salivary secretion rate $(\mathrm{mL} / \mathrm{min}) \times \operatorname{SIgA}-\mathrm{C}(\mu \mathrm{g} / \mathrm{mL})$. In addition, blood samples were taken prior to, during and after the altitude training at the first training camp. All blood samples were centrifuged $(2,000 \times \mathrm{g}, 10 \mathrm{~min})$, and serum cortisol levels that were determined by radioimmunoassay and serum IgA levels that were determined by turbid metric immunoassays were analyzed in order to compare them with the salivary component.

\section{Statistical analyses}

The obtained data were expressed as mean \pm standard error of the mean (SEM) unless otherwise stated. The values were analyzed by repeated two-way analyses of variance with sphericity tests, and, if there were significant differences, post-hoc

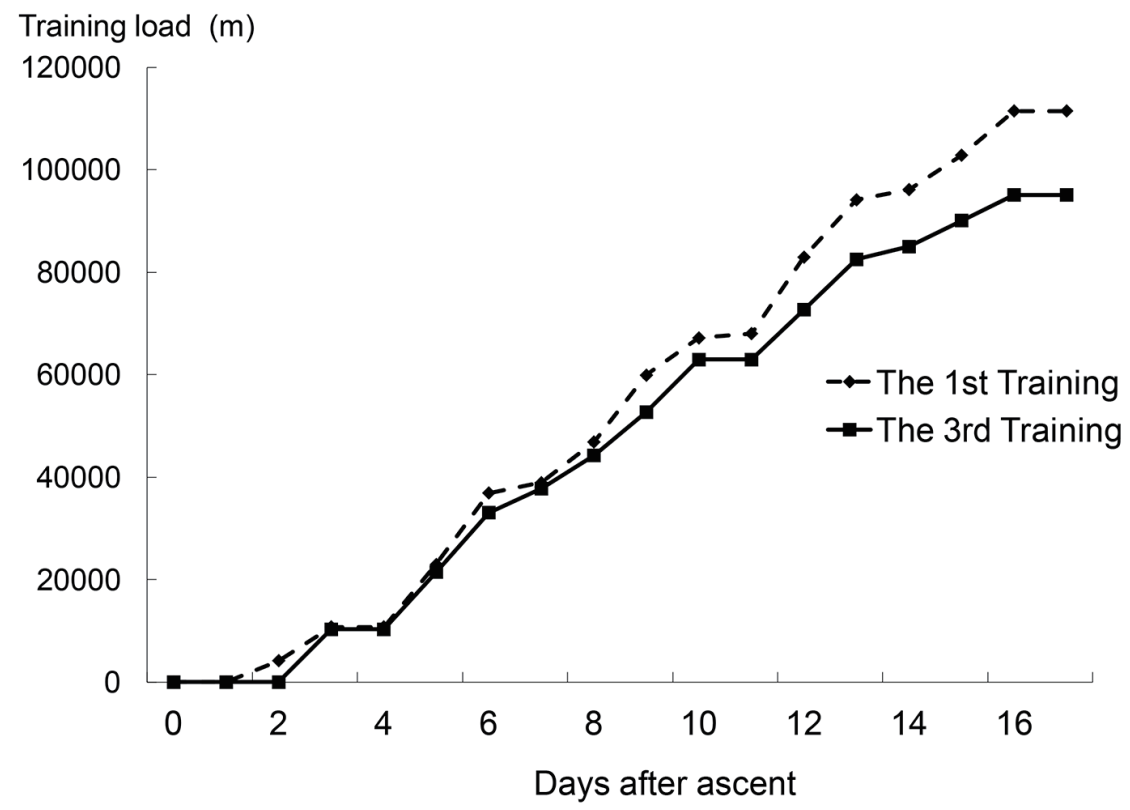

Figure 1. Cumulative training loads of the first and third training camps (all participants in each group got the same training load (m) at the certain day after ascent). 


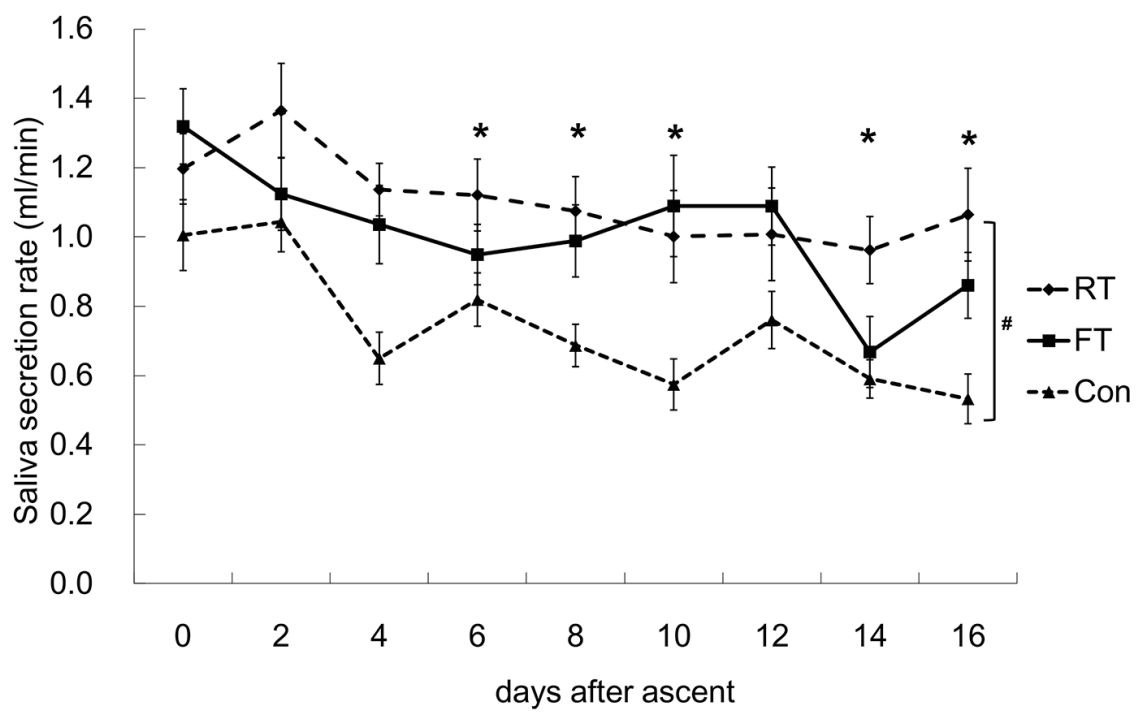

Figure 2. Changes in the salivary secretion rate (SSR). A significant difference between the repetitive training (RT) and control (Con) groups was observed. After ascent, the SSR was significantly lower from day 4 to day 16, except for day 12 compared to day 0 . ${ }^{*} \mathrm{P}<0.05$ vs. 0 time point; ${ }^{~} \mathrm{P}<0.05$ between $\mathrm{RT}$ and Con. FT: first altitude training.

pairwise comparisons were conducted using the Bonferroni method. $\mathrm{P}$ values less than 0.05 were determined to be statistically significant. These statistical analyses were conducted using the SPSS Statistics Ver. 19.0.0 program (IBM Corporation, Armonk, NY, USA, 2010).

\section{Ethics}

The research protocol was approved by the Ethical Committee of the University of Tsukuba Graduate School of Comprehensive Human Sciences Health and Physical Education.

\section{Results}

SSR, SIgA-C and SIgA-V values of the FT, RT and Con groups are shown in Figures 2-4.

As for the changes in SSR, no interactions between the dates and the groups were detected, but there was a signifi-

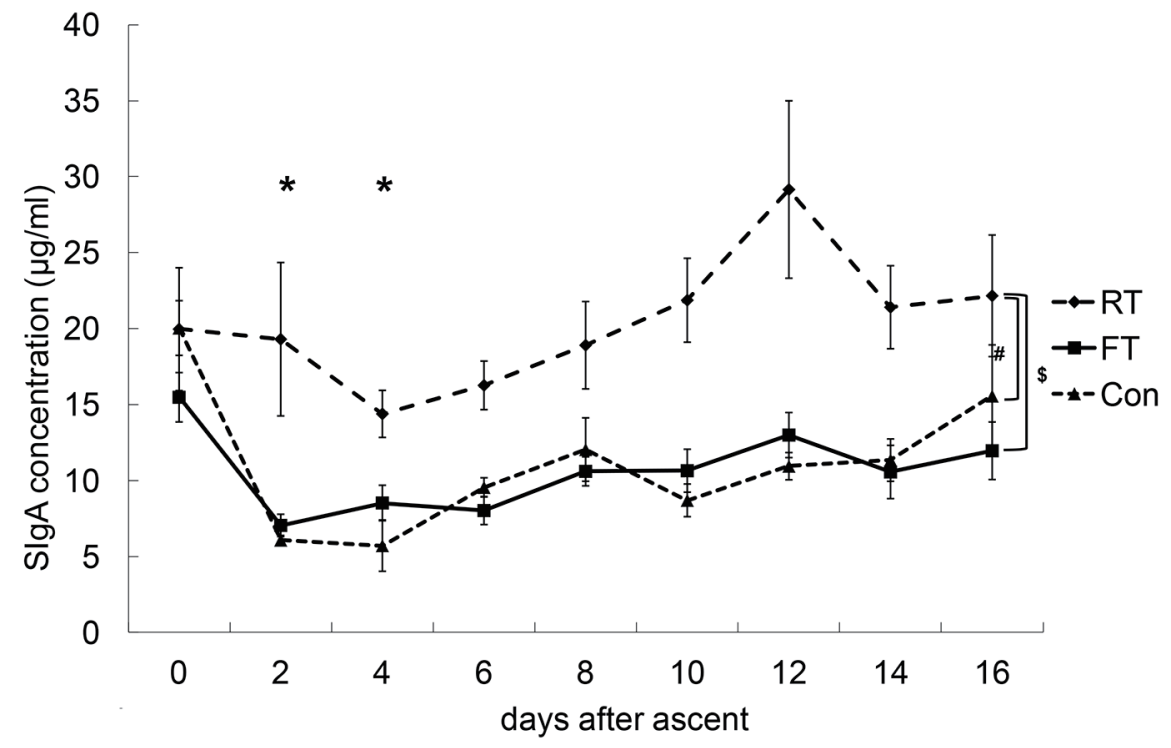

Figure 3. Changes in salivary secretory immunoglobulin A concentrations (SIgA-C). The SIgA-C of the repetitive training (RT) group was significantly higher than those of the first altitude training (FT) and control (Con) groups. The SlgA-C values at day 2 and day 4 were significantly lower compared with that at day $0 .{ }^{*} \mathrm{P}<0.05$ vs. 0 time point; ${ }^{\# P}<0.05$ between $\mathrm{RT}$ and Con; ${ }^{\$} \mathrm{P}<$ 0.05 between RT and FT. 


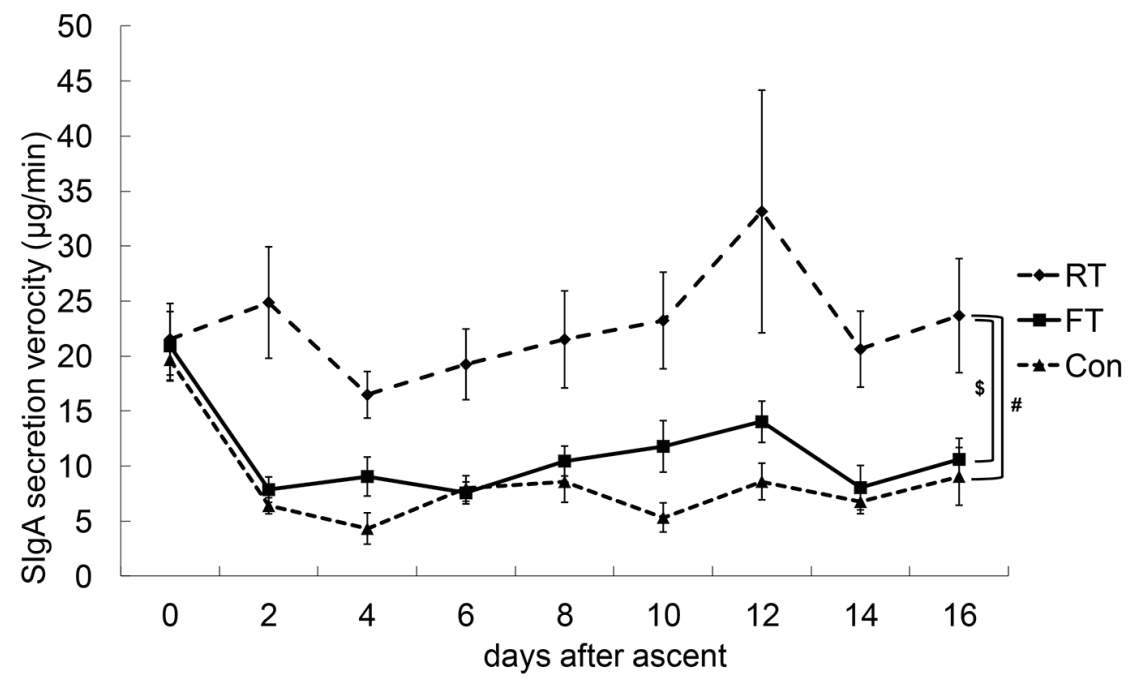

Figure 4. Changes in SIgA secretion velocity (SIgA-V). Statistically, there were no interactions between the dates and the groups with respect to SIgA-V. ${ }^{\# P}<0.05$ between repetitive training (RT) and control (Con); ${ }^{\$} \mathrm{P}<0.05$ between RT and first altitude training (FT).

cant difference between the RT and Con groups. The measured values of the groups tended to be in the order of RT $>$ FT $>$ Con. After ascent, SSR values gradually decreased and were significantly lower from day 4 to day 16 , except for day 12 compared to day 0 .

There were no interactions between the dates and the groups for the SIgA-C values. The SIgA-C value of the RT group was significantly higher than those of the FT and Con groups. Chronologically, SIgA-C values decreased immediately after ascent and gradually recovered. The SIgA-C values at day 2 and day 4 were significantly lower compared to that at day 0 , and the SIgA-C values at day 14 and day 16 were significantly higher compared to that at day 4 .

Statistically, there were no interactions between the dates and the groups for SIgA-V, and there was no chronological change, although the SIgA-V values of the RT group tended to be higher compared to those of the FT and Con groups.

\section{Discussion}

The aim of the present study was to examine the effects of altitude swim training at moderate altitudes on mucosal immunity and to examine how the repetition of the altitude trainings affected mucosal immunity. SIgA secretion from the salivary gland is the most common mucosal immune system, and SIgA has been suggested as a useful marker for athletes for evaluating their own immune conditions at the training site [6-8]. However, there have been few reports that have examined the response of SIgA during altitude training [9], and, furthermore, this is the first report that examined the changes in SIgA values caused by repetitive altitude training. We divided the subjects into three groups, namely, the first training group (FT), the experienced training group (RT) and the non training group (Con). Differences in the values with or without training could be observed by comparing the FT and Con groups. Then, the differences caused by training experience could be evaluated by comparing the FT and RT groups.

Compared to the RT and FT groups, the average age of the Con group was significantly higher. However, as far as the flow rate is concerned, the salivary glands seem to be fully developed by the age of 15 [10]. In addition, a previous study reported that children below the age of 7 years have lower salivary IgA concentrations than children above 7 years of age or adults and that there was a significant inverse relationship between saliva flow and salivary IgA concentration [11]. In the present study, all subjects were older than 15 years of age, and, thus, we believe that the differences in the ages between the groups did not influence the SSR and SIgA secretion.

As for the SSR values, there was a significant difference between the RT and Con groups, but there was no difference between the RT and FT groups. Salivary glands are innervated by both sympathetic and parasympathetic nerve fibers. Various neurotransmitters and hormones stimulate different receptors, different salivary glands and different responses $[12,13]$. One of the reasons that the SSR values of the athletes and the Con group were different was that the parasympathetic nervous system has been reported to be dominant over the sympathetic nervous system at rest in well-trained athletes like those in the RT and FT groups. One study reported that acute hypoxia ( 2 days at $4,350 \mathrm{~m}$ ) led to an increase in mean saliva flow rates both at rest and after exercise [14], while SSR values were gradually decreased and maintained at lower values several days after the ascent during the altitude training in this study. The differences in the responses might be explained by differences in the altitudes $(1,900 \mathrm{~m}$ vs. 4,350 m) in part and by other factors, such as mental and/or environmental stress. However, hypobaric-hypoxic stress induced by altitude stimulates the sympathetic nervous system. Studies from the summit of Pikes Peak $(4,300 \mathrm{~m})$ have indicated strong alpha- and beta-adrenergic components in the regulation of immune function at high altitudes that can persist for weeks after the initial 
exposure. Specifically, interleukin-6 levels are increased with acute altitude exposure, and this is primarily mediated by betaadrenergic stimulation. The levels remain increased for several weeks as a result of alpha-adrenergic activation [15].

Moreover, hemoconcentrations that are induced by dehydration were observed in the acute phase after ascent. In this study, the decreases in SSR values were significant after day 6. Dehydration by diuretic effects that occur in response to hypoxia might not be prolonged, and the decrease in SSR was not obviously influenced by dehydration.

In this study, SIgA-C values decreased immediately after ascent and then gradually recovered after day 4 . Moreover, the SIgA-C values of the RT group were consistently high than those of the FT or Con groups. These results might suggest that the SIgA-C values of the RT group were influenced by the repeated experiences with the altitude and not by hypoxic stress. While SIgA-V is determined by SSR and SIgA-C, this is represented by the actual focal mucosal immunity. In this study, SIgA-V values were not changed statistically after the ascent, while the values of the RT group were consistently higher than those of the FT and Con groups. We suggest that the SIgA-V was influenced mainly by the decrease in SIgA-C in the early phase of the altitude training (just after ascent), and, after the acute phase, it was influenced mainly by the decrease in SSR.

When the added stress of physical exercise is combined with that of hypoxia, a more pronounced impact on immune function is observed compared to that of either exercise or hypoxia alone [15], and exposure to hypobaric hypoxic stress by high altitude training can induce a decrease in SIgA [9]. Infact, exercise performed in the course of the different adventure sport activities to date was not very intense, but was very prolonged and the long time duration of the activities performed may account for the decrease of the salivary IgA levels [16].

The results of this study supported those of a study by Tiollier et al [9], in which SIgA-C decreased in the live high (above 2,500 $\mathrm{m}$ )-train low $(1,200 \mathrm{~m}$ ) group compared to the live low-train low group whose decreasing rate was about $50 \%$ compared to that before ascent. With respect to immunity in altitude sports or training, ascent to high altitudes increases the incidence of infection [17]. Bailey et al [18] reported that the occurrence of symptoms of upper respiratory tract infections or gastrointestinal infections occurred between 8 and 14 days after ascent. One of the mechanisms thought to be involved was a chronic reduction in the synthesis rate of glutamine during a long-term stay at a high altitude, which in turn may lead to gut atrophy, bacterial translocation, endotoxemia, muscle protein catabolism and a weakened immune status [19]. Our study showed a decrease in SIgA-C in the several days after ascent and a significant decrease in SSR in the 8 - 14 days after ascent. From these findings, we suggest that the acute phase of altitude training may lead to immunosuppression, including a decrease in SIgA-C, and the chronic phase may lead to the failure of focal clearance by a decrease in SSR. However, these findings highlight the potential for the misdiagnosis of altitude illness due to the similarities in nonspecific constitutional symptoms that are associated with infection and acute mountain sickness [18]. Thus, clear conclusions are difficult to make because of the overlap with the symptoms of acute mountain sickness [20]. Another reason is that most infections at high altitudes are diagnosed clinically and do not have objective data to support the diagnosis.

As for the repetitive training, a study suggested that brief daily intermittent altitude exposures at $4,300 \mathrm{~m}$ were equally as effective as continuous altitude residence in inducing physiological adaptations that are consistent with altitude acclimatization. However, this stimulus did not induce a hormonal stress response and did no harm in terms of the activation of the immune system at high altitude, as measured by white blood cell and leukocyte subset counts [21].

The results of the present study indicated that the chronological trends between the FT and RT groups were similar with respect to SSR changes. However, they were quite different for the mucosal immune index, such as the SIgA-C. Thus, we suggest that repetitive altitude training within a relatively short interval can cause the same physical stress to athletes, even though the stress of RT seemed to be less than that of FT. The differences in the FT and RT groups can be explained by other factors, such as training loads (quantity and quality), circumstances, or the individual. However, the training quantity was similar, and the quality must have been higher in the RT group than in the FT group because the RT group had the third training in order to be ready for the domestic selection for the Olympic Games. In addition, the mean age of Con group was different from those FT and RT groups warranting further researches to demonstrate any effect of age on the findings of current study.

The decreases in SIgA-C in the FT group during altitude training were remarkable compared to that of the RT group, but the trends in SIgA-C were similar. Limiting initial exposures when training or competing in adverse environmental conditions (heat, humidity, cold, altitude, or polluted air) and acclimatizing where appropriate will reduce the effects of environmental stress [22]. There are some reports that elite athletes that had engaged in high-intensity training prior to competition showed either no change or a moderate increase in cellular immune activation, such as changes in the ratio of neopterin/creatinine [23]. When we consider the conditioning of altitude training, we should pay attention to the immune condition of the athletes, regardless of their experiences with altitude training or the training interval.

\section{Conclusions}

We studied the effects of moderate altitude training and repeated experiences with such training on the response of SIgA. SIgA secretion decreased after ascent, and there were differences according to the training experience. At the acute phase, the decrease in the SIgA concentration rate was dominant. Thereafter, the decrease in the SSR was dominant. While the trend in the SIgA secretion during altitude training was similar regardless of the level of experience, we should pay attention to the athlete's physical condition as an indication of immune function during the altitude training.

\section{Acknowledgments}

We appreciate the support of the Kozuki Foundation for Sports 
and Education.

\section{Financial Disclosure}

There was no specific funding source to be mentioned.

\section{Conflict of Interest}

The authors declare that they have no conflict of interest.

\section{Informed Consent}

All subjects provided written informed consent.

\section{Author Contributions}

KW has designed and executed the study. KW has also drafted the manuscript. YM, TT, TS and YS have assisted in sample collection and analysis. SJ and ST have supervised this manuscript preparation and provided critical editing.

\section{References}

1. Bailey DM, Davies B. Physiological implications of altitude training for endurance performance at sea level: a review. Br J Sports Med. 1997;31(3):183-190.

2. Bonetti DL, Hopkins WG. Sea-level exercise performance following adaptation to hypoxia: a meta-analysis. Sports Med. 2009;39(2):107-127.

3. Garcia-Ramos A, Padial P, de la Fuente B, ArguellesCienfuegos J, Bonitch-Gongora J, Feriche B. Relationship between vertical jump height and swimming start performance before and after an altitude training camp. J Strength Cond Res. 2016;30(6):1638-1645.

4. Rodriguez FA, Iglesias X, Feriche B, Calderon-Soto C, Chaverri D, Wachsmuth NB, Schmidt W, et al. Altitude Training in Elite Swimmers for Sea Level Performance (Altitude Project). Med Sci Sports Exerc. 2015;47(9):1965-1978.

5. Bailey DM, Davies B, Romer L, Castell L, Newsholme E, Gandy G. Implications of moderate altitude training for sea-level endurance in elite distance runners. Eur J Appl Physiol Occup Physiol. 1998;78(4):360-368.

6. Gleeson M, Pyne DB. Special feature for the Olympics: effects of exercise on the immune system: exercise effects on mucosal immunity. Immunol Cell Biol. 2000;78(5):536-544.

7. Moreira A, Arsati F, Cury PR, Franciscon C, de Oliveira PR, de Araujo VC. Salivary immunoglobulin a response to a match in top-level brazilian soccer players. J Strength Cond Res. 2009;23(7):1968-1973.

8. Neville V, Gleeson M, Folland JP. Salivary IgA as a risk factor for upper respiratory infections in elite professional athletes. Med Sci Sports Exerc. 2008;40(7):1228-1236.

9. Tiollier E, Schmitt L, Burnat P, Fouillot JP, Robach P, Filaire E, Guezennec C, et al. Living high-training low altitude training: effects on mucosal immunity. Eur J Appl Physiol. 2005;94(3):298-304.

10. Crossner CG. Salivary flow rate in children and adolescents. Swed Dent J. 1984;8(6):271-276.

11. Kugler J, Hess M, Haake D. Secretion of salivary immunoglobulin A in relation to age, saliva flow, mood states, secretion of albumin, cortisol, and catecholamines in saliva. J Clin Immunol. 1992;12(1):45-49.

12. Culp DJ, Graham LA, Latchney LR, Hand AR. Rat sublingual gland as a model to study glandular mucous cell secretion. Am J Physiol. 1991;260(6 Pt 1):C1233-1244.

13. Humphrey SP, Williamson RT. A review of saliva: normal composition, flow, and function. J Prosthet Dent. 2001;85(2):162-169.

14. Pilardeau P, Richalet JP, Bouissou P, Vaysse J, Larmignat P, Boom A. Saliva flow and composition in humans exposed to acute altitude hypoxia. Eur J Appl Physiol Occup Physiol. 1990;59(6):450-453.

15. Mazzeo RS. Altitude, exercise and immune function. Exerc Immunol Rev. 2005;11:6-16.

16. Teixeira AM, Santos AC, Oliveira M, Rosado MF, Martins M, Cunha MR. Salivary IgA response during one week of adventure sports activities with altitude exposure. Annals of Research in Sport and Physical Activity. 2011(2):12-20.

17. Demel U, Domej W, Tilz GP. [Function and repertoire of the immune system in body's defense]. Wien Med Wochenschr. 2000;150(8-9):175-177.

18. Bailey DM, Davies B, Castell LM, Collier DJ, Milledge JS, Hullin DA, Seddon PS, et al. Symptoms of infection and acute mountain sickness; associated metabolic sequelae and problems in differential diagnosis. High Alt Med Biol. 2003;4(3):319-331.

19. Wagenmakers AJ. Amino acid metabolism, muscular fatigue and muscle wasting. Speculations on adaptations at high altitude. Int J Sports Med. 1992;13(Suppl 1):S110113.

20. Walsh NP, Whitham M. Exercising in environmental extremes : a greater threat to immune function? Sports Med. 2006;36(11):941-976.

21. Beidleman BA, Muza SR, Fulco CS, Cymerman A, Staab JE, Sawka MN, Lewis SF, et al. White blood cell and hormonal responses to $4300 \mathrm{~m}$ altitude before and after intermittent altitude exposure. Clin Sci (Lond). 2006;111(2):163-169.

22. Pyne DB, Gleeson M, McDonald WA, Clancy RL, Perry $\mathrm{C}$, Jr., Fricker PA. Training strategies to maintain immunocompetence in athletes. Int J Sports Med. 2000;21(Suppl 1):S51-60.

23. Jakeman PM, Weller A, Warrington G. Cellular immune activity in response to increased training of elite oarsmen prior to Olympic competition. J Sports Sci. $1995 ; 13(3): 207-211$. 